\title{
Stochastic Evaluation of Maximum Wind Installation in a Radial Distribution Network
}

\author{
Peiyuan Chen, Member, IEEE, Birgitte Bak-Jensen, Member, IEEE, and Zhe Chen, Senior Member
}

\begin{abstract}
This paper proposes an optimization algorithm to find the maximum wind installation in a radial distribution network. The algorithm imposes a limit on the amount of wind energy that can be curtailed annually. The algorithm implements the wind turbine reactive power control and wind energy curtailment using sensitivity factors. The optimization is integrated with Monte Carlo simulation to account for the stochastic behavior of load demand and wind power generation. The proposed algorithm is tested on a real $20 \mathrm{kV}$ Danish distribution system in Støvring. It is demonstrated that the algorithm executes reactive compensation and energy curtailment sequentially in an effective and efficient manner.
\end{abstract}

Index Terms-Stochastic, wind power, optimization, distribution

\section{INTRODUCTION}

The evaluation of maximum wind installation in a distribution network is a goal that concerns several parties involved in wind energy business [2], [2]. From the perspective of wind power producers, this may increase their annual profits from energy trading. From the perspective of distribution network operators, this indicates that how much wind energy their network can accommodate before considering network reinforcement. From the perspective of the environment and society, this goal complies with $\mathrm{CO} 2$ reduction and sustainable development.

Certain research effort has been devoted to the maximization of wind installation in a distribution network [2]-[4]. The focus is mainly on the use of active management schemes for the voltage control. The optimization algorithm is based on multi-period optimal power flow [2], [3], and a linear optimal power flow [4]. However, none of the work imposes a limitation on the amount of annual wind energy curtailment.

This paper aims to find the maximum wind turbine (WT) capacity that can be installed in a network allowing for a specific amount of wind energy curtailment. Furthermore, the paper also identifies different contributions from WT reactive power control and energy curtailment to maximizing WT

This work was supported by the Danish Agency for Science Technology and Innovation, under the project of 2104-05-0043.

P. Chen, B. Bak-Jensen and Z. Chen are all with the Department of Energy Technology, Pontoppidanstraede 101, Aalborg University, Aalborg, 9220 Denmark (e-mail: pch@et.aau.dk, bbj@et.aau.dk, zch@et.aau.dk).

P. Chen is also with the Division of Electric Power Engineering, Hörsalsvägen 11, Chalmers University of Technology, Gothenburg, 41296 Sweden (e-mail: peiyuan@chalmers.se). capacity in the network. The evaluation considers the stochastic behavior of load demand and wind power generation (WPG) based on time series measurement.

\section{Evaluation ON A Single CABle AND A RADiAl FEEDER}

\section{A. Evaluation on a Single Cable}

The capability of a cable to transmit power is constrained by the current and voltage limit requirements of the cable. Thus, a cable has a maximum intake of wind power. Furthermore, the maximum wind power transmitted also depends on the power factor setting or the reactive power output of the WT. Such a maximization issue can be formulated as a standard nonlinear constrained optimization problem [5].

Simulations are carried out on a three-core $120 \mathrm{~mm}^{2}$ cable with a measured hourly wind power time series over a year. The cable length varies from $0.1 \mathrm{~km}$ to $60 \mathrm{~km}$ at a step of 0.1 $\mathrm{km}$. Fig. 1 shows the maximum wind power of the cable under three different power factor settings: 1) unity power factor $(\tan \phi=0), 2)$ power factor no less than $0.8(|\tan \phi| \leq 0.75)$, and 3) any power factor values $(|\tan \phi| \leq \infty)$. According to the figure, as the power factor range is widened, the maximum wind power is increased. For instance, for a $40 \mathrm{~km}$ cable, the maximum wind power of the cable is $1.8 \mathrm{MW}$ when $\tan \phi=0$, but is increased to $2.4 \mathrm{MW}$ when $|\tan \phi| \leq 0.75$ and to 5.6 MW when $|\tan \phi| \leq \infty$. The dominant limiting factor is the maximum voltage. Consequently, for a given capacity of wind installation, an inductive power factor setting brings down the voltage. In other words, a proper power factor setting can increase the maximum wind power intake of a cable.

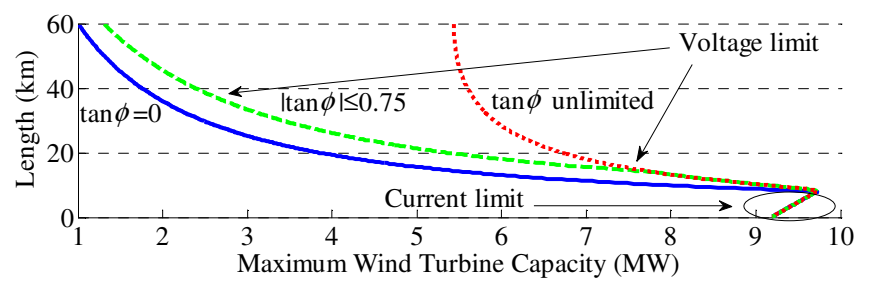

Fig. 1 Maximum wind power of a $20 \mathrm{kV} 120 \mathrm{~mm}^{2}$ three-core cable under different power factor values for different cable length

Another way to increase the annual wind energy production is to allow a certain amount of energy curtailment. This can increase the installed WT capacity, which leads an increase in 
the total wind energy harvest. The relation between the annual generated wind energy $E$ and the installed WT capacity $P_{r}$ can be calculated by:

$$
E=C_{\mathrm{f}} \cdot 8760 \cdot P_{\mathrm{r}} \cdot\left(1-C_{\mathrm{E}}\right),
$$

where $C_{\mathrm{f}}$ is the capacity factor of the WPG; $P_{\mathrm{r}}$ is the installed capacity of the WT; $C_{\mathrm{E}}$ is the allowed energy curtailment in percentage. The capacity factor of the wind power time series is 0.25 .

In the case when $C_{\mathrm{E}}=0$, which is no energy curtailment, the maximum WT capacity can be determined using Fig. 1. In the case when $C_{\mathrm{E}}>0$, the maximum WT capacity depends on the probability distribution of the wind power.

Fig. 2 shows the cumulative distribution function of a measured wind power time series. If $C_{\mathrm{E}}=5 \%$, then the corresponding wind power is 0.812 p.u. Thus, the maximum WT capacity is increased to $123 \%(=1 / 0.812)$. Consequently, according to (1), the annual generated wind energy $E$ rises to $117 \%(=123 \% \times(1-5 \%))$ of the value in the case when no energy curtailment is allowed. For this particular wind power time series, Fig. 3 shows the increased percentage of annual wind energy and installed WT capacity when the annual wind energy is curtailed from 0 to $20 \%$. The figure shows that the annual wind energy has a slower increment than the installed WT capacity.

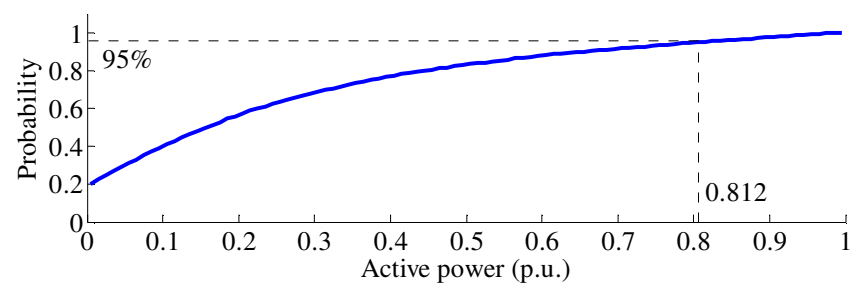

Fig. 2. Cumulative distribution function of a measured wind power time series.

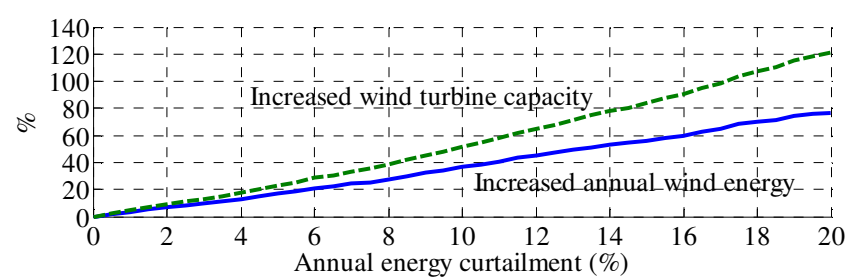

Fig. 3 Increased annual wind energy and wind turbine capacity due to annual energy curtailment.

\section{B. Evaluation on a Radial Feeder}

Normally, WTs are distributed along a radial feeder as shown in Fig. 4. According to Fig. 1, when the voltage limit is the dominant constraint, the maximum WT capacity increases as the cable length decreases. Therefore, in order to maximize the total WT capacity on the feeder, all the WTs should be connected to as close as possible to the grid, e.g., bus 1 . In this case, the method described in section II can be used to determine the maximum WT capacity. On the other hand, when the current limit becomes the main constraint, the maximum WT capacity increases as the cable length increases.
This is because a longer cable induces higher voltage rise, which causes a lower current at a fixed power injection. This makes the optimal allocation of WTs less straightforward than the case with voltage limit constraint. The solution can also be based on a similar optimization algorithm. For instance, for the radial feeder shown in Fig. 4, assume that $n=2, L_{1}=L_{2}$, and the cable is three-core with conductor size of $120 \mathrm{~mm}^{2}$. For such a two-section feeder, the maximum WT capacity at bus $1\left(P_{r 1}\right)$ and bus $2\left(P_{r 2}\right)$ are presented in Fig. 5 . When the feeder length is longer than $16 \mathrm{~km}$, the main constraint is the voltage limit (1.06 p.u.) at bus 2 . Thus, all the wind power is injected to bus 1 ( $P_{r 1}$ is maximum) and $P_{r 2}$ is zero. When the feeder length is shorter than $16 \mathrm{~km}$, the current limit of section $L_{1}$ becomes the dominant constraint. Consequently, more and more wind power is injected into bus 2 so that the current through section $L_{1}$ is reduced as the voltages at bus 1 and bus 2 rises. When the feeder length decreases even further $(\leq 10.7$ $\mathrm{km}$ ), the voltage at bus 2 drops rapidly, which leads to a slight cutback of $P_{r 2}$.

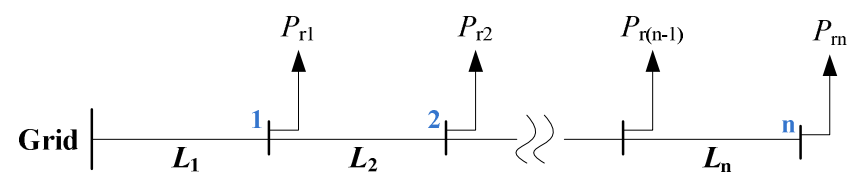

Fig. 4. WTs connected to a radial feeder of a cable.

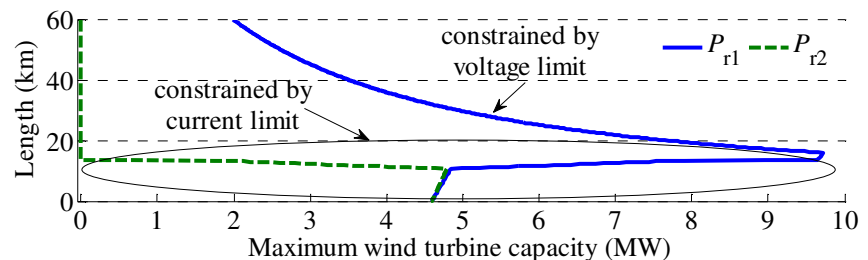

Fig. 5. Maximum wind turbine capacity at bus 1 and bus 2 of a two-section feeder.

It is also not complicated to evaluate the maximum WT capacity of the feeder if involving reactive power control of the WTs. Similar curves to the ones in Fig. 5 can be obtained. Furthermore, if energy curtailment is also considered, for the case when the dominant constraint is voltage limit, the solution is to curtail the wind energy from the WTs connected at the end of the feeder first. However, this is not the case when the dominant constraint is current limit. The optimal solution is not to cut for example 5\% of wind energy at both WT locations as the maximum WT capacity of the feeder does not increase linearly with respect to the WT capacity at each location.

Moreover, in the case when the feeder is mixed with load demand and WPG as shown in Fig. 6, the optimal solution also becomes more complex. The reason is that the load demand at bus 1 causes a forward voltage drop, which varies as the load fluctuates. This voltage drop compensates the voltage rise caused by the wind power at bus 2 . Consequently, the voltage at bus 2 is not necessarily the highest when the WT generates at its rated power as it also depends on the voltage drop at bus 1 . In other words, if the strategy of energy curtailment is employed, it is not necessarily to curtail the top $5 \%$ wind energy as discussed in section II. In this case, in 
order to evaluate maximum WT capacity, a more detailed analysis is required to include both the stochastic load variation and wind power variation. Such an analysis may require another optimization process for the energy curtailment.

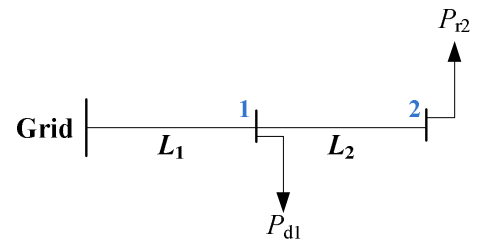

Fig. 6. A two-section feeder with load demand and wind power generation.

\section{Proposed Optimization StruCture}

This paper proposes an optimization structure as shown in Fig. 7. It mainly consists of two optimization loops: the outer loop maximizes the total WT capacity and the inner loop minimizes the energy curtailment while fulfilling the technical constraints through a constrained load flow algorithm [6]. In order to account for the stochastic variation of WPG and load when curtailing the wind energy, a Monte Carlo simulation is adopted to carry out the inner-loop optimization. The following section introduces the constrained load flow algorithm based on sensitivity analysis [6]-[8].

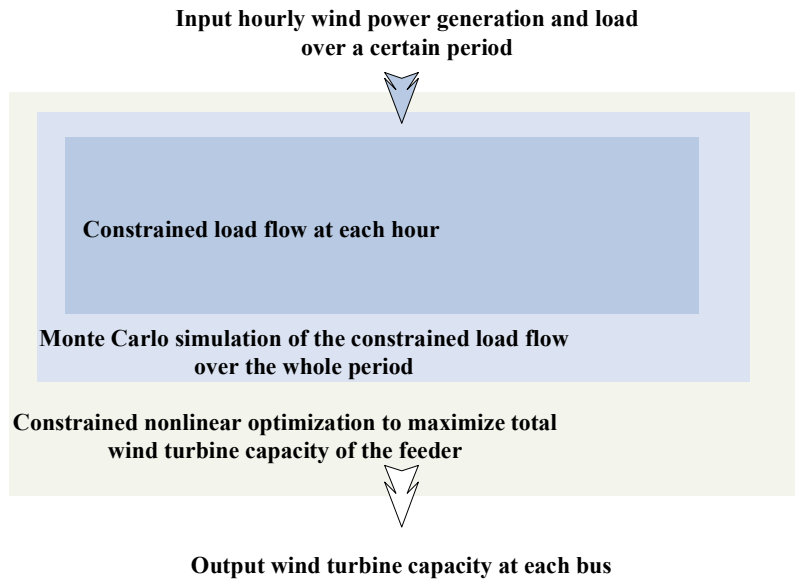

Fig. 7. Proposed solution to finding the maximum wind turbine capacity of a feeder considering energy curtailment based on constrained load flow.

\section{CONSTRAINED LOAD FLOW BASED ON SENSITIVITY ANALYSIS}

Fig. 8 shows the flow chart of the constrained load flow algorithm. The constrained load flow algorithm implements three main subroutines into the standard Newton-Raphson based load flow algorithm. The main purpose of these subroutines is to constrain the bus voltages and the line currents by regulating the WT power output. The first subroutine checks if the lower limit of the voltage is violated. In the case of undervoltage, the reactive power control of the WT is activated. The second subroutine checks if the upper limit of the voltage is violated. In the case of overvoltage, the reactive power control is activated first. If the reactive power output reaches its limit, then the active power control takes over by curtailing wind power. The third subroutine checks if overcurrent through a line occurs. In this case, the strategy of wind power curtailment is applied.

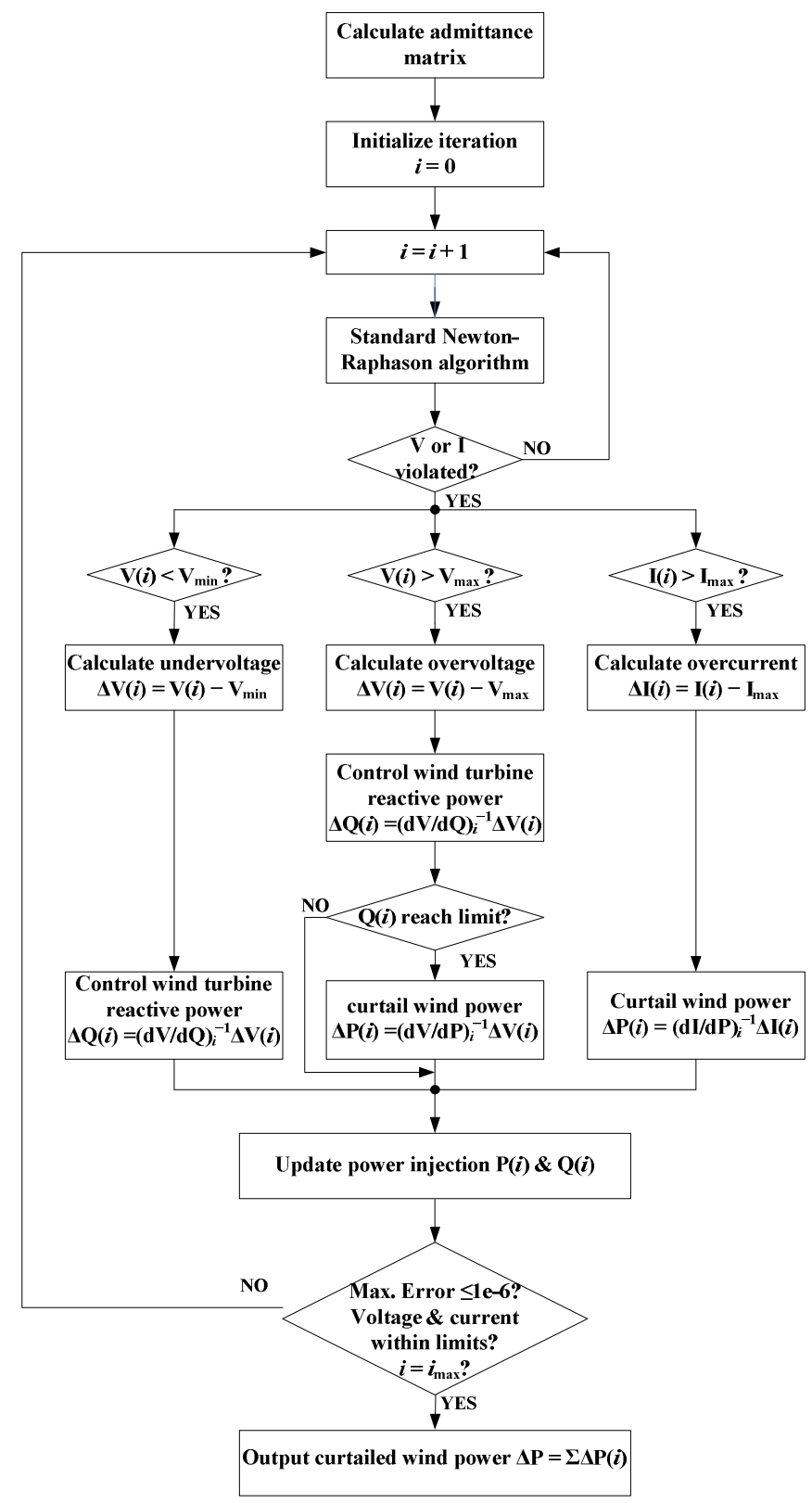

Fig. 8. Flow chart of constrained load flow algorithm with reactive power control and energy curtailment of WT.

The sensitivity factor $\mathrm{d} V / \mathrm{d} Q$ and $\mathrm{d} V / \mathrm{d} P$ can be obtained by linearizing the load flow equation :

$$
\left[\begin{array}{c}
\Delta \delta \\
\Delta V
\end{array}\right]=\mathbf{J}^{-1}\left[\begin{array}{l}
\Delta \mathbf{P} \\
\Delta Q
\end{array}\right]
$$

where $\mathbf{J}$ is the Jacobian matrix; $\Delta \boldsymbol{\delta}$ and $\Delta \mathbf{V}$ are the change in voltage angle and magnitude, respectively; $\Delta \mathbf{P}$ and $\Delta \mathbf{Q}$ are the change in active power and reactive power injection, respectively. Thus, the sensitivity factor $\mathrm{d} V / \mathrm{d} Q$ and $\mathrm{d} V / \mathrm{d} P$ are 
the corresponding row of the inverted Jacobian matrix. As the current magnitude is defined as:

$I_{i}=\sqrt{P_{i}^{2}+Q_{i}^{2}} / V_{i}$

The sensitivity factor $\mathrm{d} I / \mathrm{d} P$ can be approximated by:

$d I_{i} / d P_{i} \approx P_{i} /\left(V_{i} \sqrt{P_{i}^{2}+Q_{i}^{2}}\right)$

The sensitivity factor in (4) is close to 1 , if the reactive power output is much less than the active power output.

\section{OPTIMAL CONSTRAINED LOAD FLOW}

The algorithm of the optimization procedures described in Fig. 7 (b) is presented in Fig. 9.

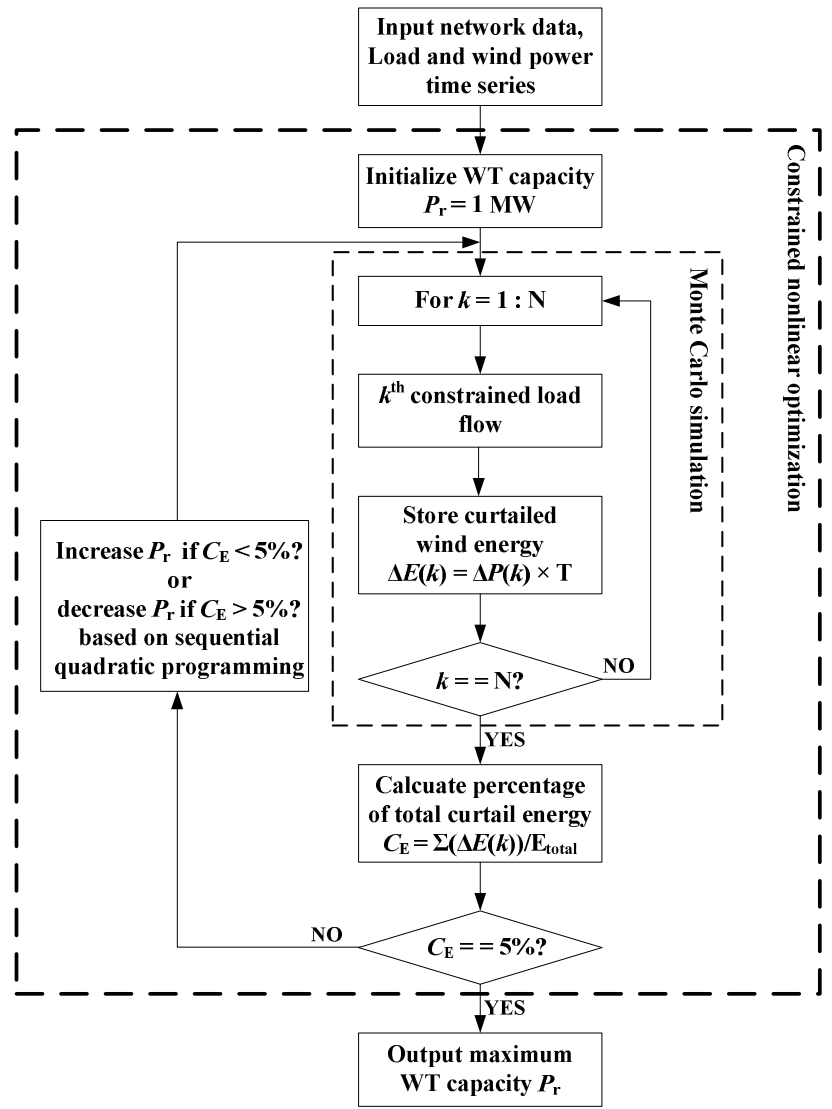

Fig. 9. Flow chart of the proposed algorithm for finding maximum wind turbine capacity of the network.

The algorithm combines constrained load flow and constrained nonlinear optimization. The algorithm requires inputs of wind power and load time series and provides outputs in the form of maximum WT capacity in a feeder or network. The algorithm mainly consists of two parts. The first part is the Monte Carlo simulation of the constrained load flow that is carried out for each time step of the time series data. The aim of the first part is to constrain the bus voltages and line currents within the required limits by regulating the reactive and active power output of the WTs. The second part is the constrained nonlinear optimization based on sequential quadratic programming [10]. The objective of this part is to find the maximum WT capacity in the network while allowing a given amount of annual energy curtailment. In the following section, the $20 \mathrm{kV}$ Støvring distribution system is used to demonstrate the proposed algorithm.

\section{CASE STUDY: STøVRING 20 KV DistRIBUTION SYSTEM}

\section{A. Network Description}

Fig. 10 shows the modified configuration of the Støvring distribution system. There are two main changes to the existing system. The first one is that the substation is planning to supply a new load area, which is $5 \mathrm{~km}$ away. In the new load area, new WTs are also planned. The second one is that the old WTs connected to the end of the SØRP feeder are to be replaced by new WTs. The capacities of all the new WTs are to be decided. On the basis of such a network, the following subsection demonstrates the simulation results from the optimal constrained load flow algorithm proposed in Fig. 9.

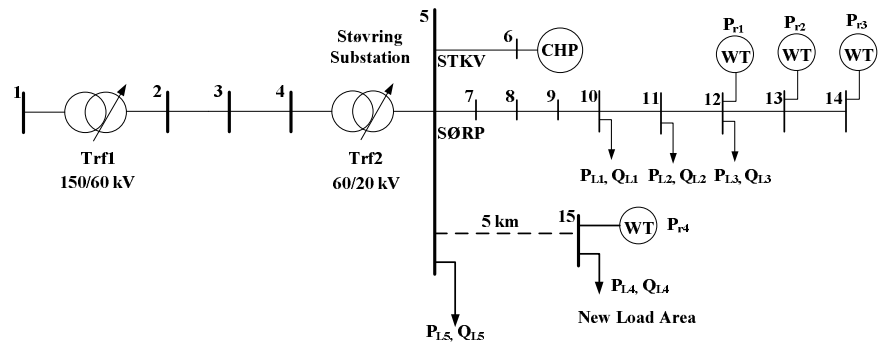

Fig. 10. Modified network configuration of the Støvring distribution system.

\section{B. Monte Carlo simulation with constrained load flow}

According to Fig. 9, the constrained nonlinear optimization provides inputs of the WT capacities to the Monte Carlo simulation of the constrained load flow. Assume that the WT capacity connected to the SØRP feeder is $3 \mathrm{MW}$ each and the one connected to the new load area is $12 \mathrm{MW}$. The cable that supplies the new load area is a three-core cable with a conductor size of $120 \mathrm{~mm}^{2}$. All the WTs can regulate the power factor within $[0.95,1]$ in both leading and lagging direction. The maximum allowed voltage is set to 1.06 p.u. The current rating of the $120 \mathrm{~mm}^{2}$ cable is $265 \mathrm{~A}$.

A Monte Carlo simulation of the constrained load flow is carried out on the network. Fig. 11 (a) shows the time series of the voltage at bus 14 . The voltage is constrained under the maximum limit 1.06 p.u. Fig. 11 (b) shows the corresponding cumulative distribution function of the voltage, where around $15 \%$ of the time, the voltage is around 1.06 p.u. This indicates that overvoltage may occur if the reactive or active power control is not active. 


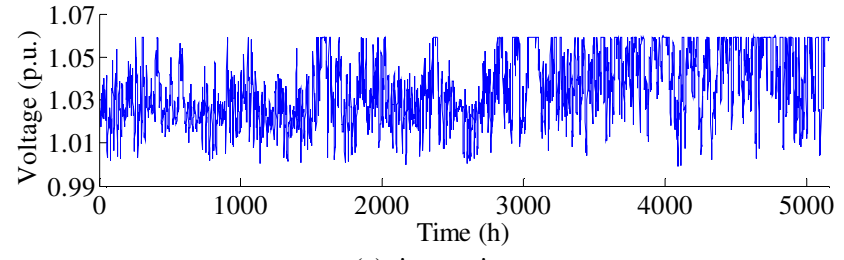

(a) time series

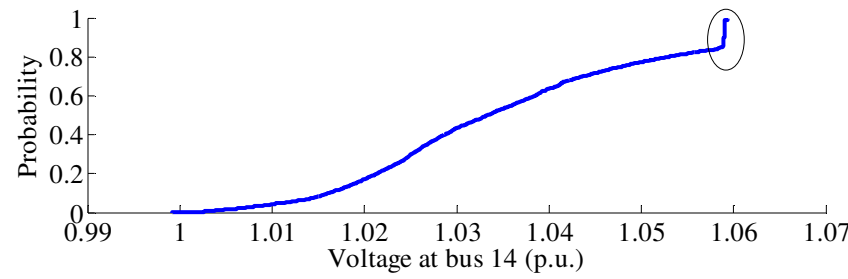

(b) cumulative distribution function

Fig. 11 Voltage magnitude at bus 14.

Fig. 12 shows the reactive power of the WT at bus 14 . The reactive power is expressed in percentage of the WT capacity ( $3 \mathrm{MW}$ ). The negative value indicates that the WT absorbs reactive power to keep the bus voltage within limits. For around $15 \%$ of the time, the reactive power output of the WT is higher than $2 \%(0.06 \mathrm{MVAr})$. For the remaining $85 \%$ of the time, the reactive power output of the WT is close to but not exactly zero. This is because the reactive power control is already activated from the $2^{\text {nd }}$ iteration of the constrained load flow algorithm. Even though the steady-state voltage may be lower than 1.06 p.u., the voltage at the early iterations may still be higher than 1.06 p.u. as the algorithm has not converged yet. However, this does not affect the effectiveness of the algorithm as a small amount of reactive power absorption only brings down the voltage slightly. Furthermore, as shown in Fig. 12 (b), for around $1 \%$ of the time, the reactive power absorption of the WT reaches its minimum value (0.99 MVAr). In this case, the active power control of the WT is activated. Fig. 13 shows the corresponding active power reduction when the reactive power control reaches its limit. A maximum reduction of $25 \%(0.75 \mathrm{MW})$ occurs during the whole period. However, the total energy curtailed during the whole period is only $0.6 \%$ (22.6 MWh).

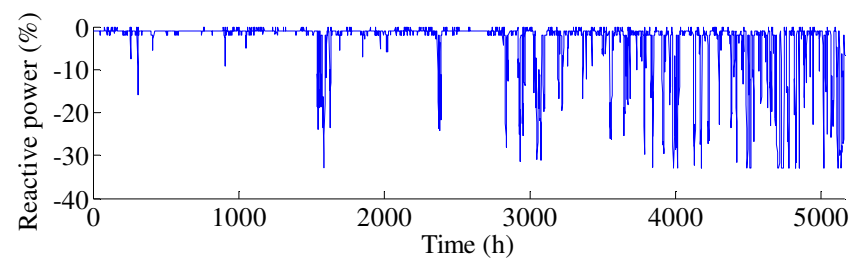

(a) time series

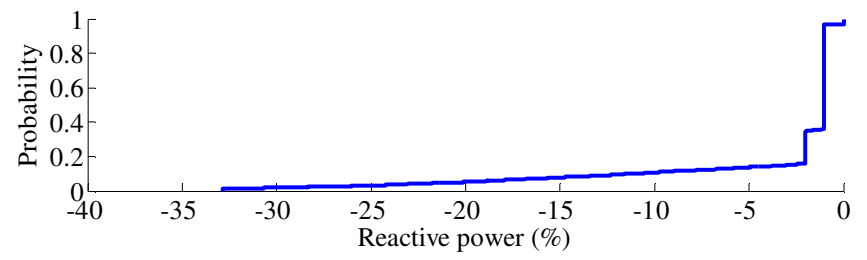

(b) cumulative distribution function

Fig. 12 Reactive power absorption of the WT at bus 14.

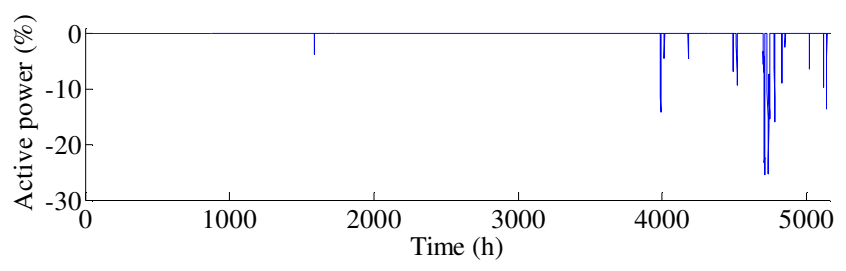

Fig. 13 Active power reduction of the WT at bus 14 .

Fig. 14 (a) shows the current through the $5 \mathrm{~km}$ cable connecting the new load area. The current rating of the cable is 0.0918 p.u. (265 A). Overcurrent occurs for around $1 \%$ of the time. In the case of overcurrent, only the active power control is activated. Fig. 14 (a) shows the corresponding active power reduction of the WT at bus 15 . A maximum reduction of $12 \%$ (1.44 MW) occurs during the whole period. However, the total energy curtailed during the whole period is only $0.3 \%$ (42.2 MWh).

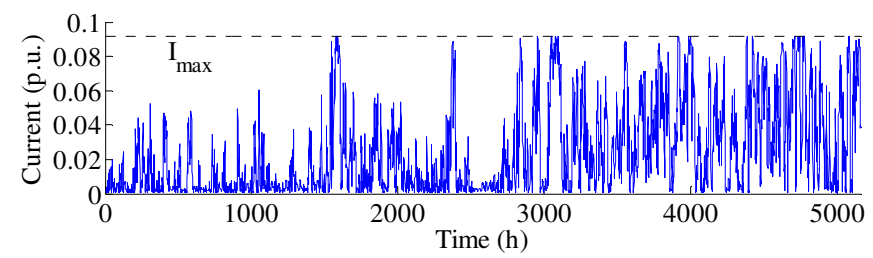

(a) current through the $120 \mathrm{~mm} 2$ three-core cable

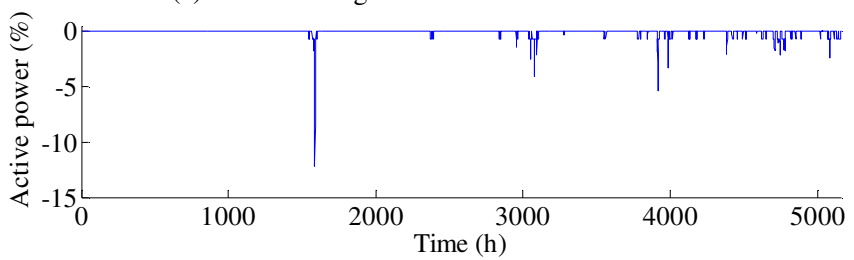

(b) active power reduction of the WT at bus 15 .

Fig. 14 Current and active power in new load area.

\section{Optimal Constrained Load Flow}

According to Fig. 9, the maximum WT capacity of the distribution network can be evaluated by implementing a constrained nonlinear optimization on top of the Monte Carlo simulation. Such a combined optimization approach not only takes into account the stochastic behavior of load demand, WPG, and CHP generation, but also ensures that the energy curtailment strategy is executed under necessary conditions such as during high generation and low demand period.

It is assumed that the three WTs connected to the SØRP feeder have the same capacity and are controlled simultaneously. However, the WT in the new load area is controlled individually. It is also assumed that the substation transformer is large enough to transport the maximum reverse power flow.

Table I summarizes the maximum WT capacity of the network for five different cases. Case 1 is the base case, which does not implement the reactive and active power control strategies. In this case, the maximum capacity is $1.3 \mathrm{MW}$ for each WT connected to the SØRP feeder, and 10.7 MW for the WT at bus 15. It is the voltage limit that stops the increment of the WT capacity at bus 12,13 and 14 . In contrast, it is the current limit that prevents the growth of the WT capacity at 
bus 15 . In case 2, when $5 \%$ wind energy curtailment is allowed, the maximum WT capacity at bus 14 is increased by around $15 \%$, while the maximum WT capacity at bus 15 is increased almost by $50 \%$. Case 3 further allows the WT power factor to be between 0.95 and 1 . This raises the maximum WT capacity at the SØRP feeder by almost three times. However, the power factor of the WT at bus 5 remains around 1 in order to minimize the current flow in the network. It can be seen from the first three cases that the energy curtailment strategy is more effective to increase the maximum WT capacity if the restricting factor is the current limit, whereas the reactive power control strategy is more effective if the restricting factor is the voltage limit. In case 5, the minimum power factor value is extended to 0.9 . In this case, the maximum WT capacity at SØRP is increased by another $28 \%$. The last case uses a 240 $\mathrm{mm} 2$ cable instead of the $120 \mathrm{~mm} 2$ cable for supplying the new load area. Compared to case 2, the maximum WT capacity at bus 15 is increased by $57 \%$. This is expected as a thicker cable has a higher current rating. Thus, a thicker cable can be a good choice if more WTs are expected to be built in the future. For this particular network, the simulation time of each case is around 12 to $20 \mathrm{~min}$.

TABLE I

MAXIMUM WT CAPACITY BASED ON THE OPTIMAL CONSTRAINED LOAD FLOW ALGORITHM.

\begin{tabular}{|c|c|c|c|c|c|}
\hline \multirow[t]{2}{*}{ Case } & \multirow{2}{*}{$\begin{array}{c}\text { Cable } \\
\text { size } \\
\left(\mathrm{mm}^{2}\right)\end{array}$} & \multirow{2}{*}{$\begin{array}{l}\text { Minimum } \\
\text { power } \\
\text { factor }\end{array}$} & \multirow{2}{*}{$\begin{array}{c}\text { Curtailed } \\
\text { wind } \\
\text { energy }\end{array}$} & \multicolumn{2}{|c|}{$\begin{array}{c}\text { Maximum WT capacity } \\
(\mathrm{MW})\end{array}$} \\
\hline & & & & $P_{\mathrm{r} 1}=P_{\mathrm{r} 2}=P_{\mathrm{r} 3}$ & $P_{\mathrm{r} 4}$ \\
\hline 1 & 120 & 1 & 0 & 1.3 & 10.7 \\
\hline 2 & 120 & 1 & $5 \%$ & 1.5 & 15.9 \\
\hline 3 & 120 & 0.95 & $5 \%$ & 4.3 & 15.8 \\
\hline 4 & 120 & 0.90 & $5 \%$ & 5.5 & 15.7 \\
\hline 5 & 240 & 1 & $5 \%$ & 1.4 & 25.0 \\
\hline
\end{tabular}

\section{CONCLUSIONS}

This paper has successfully proposed an optimization algorithm to identify the maximum wind installation in a radial distribution network when considering the voltage and current limit of the network. It was pointed out that the solution is straightforward if the network contains only wind turbines. However, in a network with a mixture of loads and wind turbines, a non-linear optimization framework is required to find the maximum wind installation. Furthermore, the reactive power control and wind energy curtailment of wind turbines are implemented using sensitivity factors, which are incorporated into the optimization algorithm. The reactive power control is the first option and has a greater impact on maximum wind installation when the dominant limiting factor is the maximum or minimum voltage. In contrast, wind energy curtailment is the second option, which plays a more important role when the dominant limiting factor is the maximum current.

\section{REFERENCES}

[1] G. P. Harrison, P. Siano, A. Piccolo and A. R. Wallace, "Exploring the trade-offs between incentives for distributed generation developers and DNOs," IEEE Trans. Power Systems, vol. 22, pp. 821-828, 2007.
[2] P. Siano, P. Chen, Z. Chen and A. Piccolo, "Evaluating maximum wind energy exploitation in active distribution networks," IET Proc. Gener. Transm. \& Distrib., vol. 4, pp. 598-608, 2010.

[3] L. F. Ochoa, A. Keane, C. Dent and G. P. Harrison, "Aplying active network management schemes to an irish distribution network for wind power maximisation," in Proc. $20^{\text {th }}$ International Conference on Electricity Distribution (CIRED), 2009.

[4] S. N. Liew and G. Strbac, "Maximising penetration of wind generation in existing distribution networks," IET Proc. Gener. Transm. \& Distrib., vol. 149, pp. 256-262, 2002

[5] J. Arora, Introduction to Optimum Design. (2nd ed.). London: Elsevier Academic Press, 2004

[6] T. S. Karakatsanis and N. D. Hatziargyriou, "Probabilistic constrained load flow based on sensitivity analysis," IEEE Trans. Power Systems, vol. 9, pp. 1853-1860, 1994.

[7] N. D. Hatziargyriou, "Probabilistic constrained load flow for optimizing generator reactive power resouces," IEEE Trans. Power Systems, vol. 15, pp. 687-693, 2000.

[8] N. D. Hatziargyriou and T. S. Karakatsanis, "A probabilistic approach to control variable adjustment for power system planning applications," International Conference on Control, vol. 1, pp.733-738 Mar. 1994

[9] H. Saadat, Power System Analysis. (3rd ed.). USA: PSA Publishing, 2010

[10] Mathworks, MATLAB Optimization Toolbox TM4: User's Guide. Natick, MA, 2009 